\title{
Tinea hidden by a vemurafenib-induced phototoxic reaction in a patient with metastatic melanoma taking dexamethasone
}

\section{Clinical record}

A 41-year-old man with stage IV BRAF-V600E (valine replaced with glutamic acid at amino acid position 600 in the BRAF kinase) metastatic melanoma was started on vemurafenib therapy on a compassionate access program. Before this, he had been on long-term dexamethasone therapy ( $4 \mathrm{mg}$ daily) for management of brain oedema related to multiple brain metastases. With the exception of his cutaneous melanoma, he had no past history of dermatological conditions, including tinea corporis and photosensitivity.

One week after starting vemurafenib therapy $(960 \mathrm{mg}$ twice daily), the patient developed a severe drug-induced photosensitivity reaction, with blistering and erosions on sunexposed areas of skin. Despite implementing adequate sun avoidance measures and using topical corticosteroids in the acute setting, minimal improvement was seen. As a result, the daily dose of dexamethasone was increased to $8 \mathrm{mg}$ daily for the next 3 weeks and then tapered back down.

Nine months later, the patient was still taking vemurafenib $960 \mathrm{mg}$ twice daily and the dexamethasone dosage had been tapered to $4 \mathrm{mg}$ daily. Although the vemurafenib-induced photosensitivity reaction had ameliorated, persistent blistering, erosions and erythema were noted on the dorsum of both hands (Figure, A). One month later, several new lesions developed on the left forearm, including an annular erythematous plaque with a scaly surface, scattered pustules and ill defined borders. A similar lesion on the left side of the chest was also identified. Skin scrapings were collected and sent for culture. Trichophyton rubrum was identified and the patient was diagnosed with tinea corporis. He was prescribed topical antifungal cream (clotrimazole every 8 hours) for these lesions.

After 7 weeks of clotrimazole therapy, the patient reported a slight reduction in the erythema and scaling of the forearm and chest lesions. However, multiple new pustules had developed on the hands and face in addition to several erythematous nodules over the dorsum of the hands and forearms (Figure, B) suggestive of Majocchi granuloma. A medium-sized inflammatory mass was also identified on the right cheek with multiple pustules, crusts and excoriations and a purulent discharge from follicular orifices (Figure, C), in keeping with facial kerion celsi.

Given the long-term dexamethasone use, previous diagnosis of T. rubrum infection and ongoing progression of the infection despite topical antifungal therapy, the patient was prescribed oral itraconazole, $200 \mathrm{mg}$ daily, for 6 weeks. The kerion celsi was managed with multiple incisions and drainages. Reduced severity of all the skin lesions was subsequently seen over the course of the therapy.
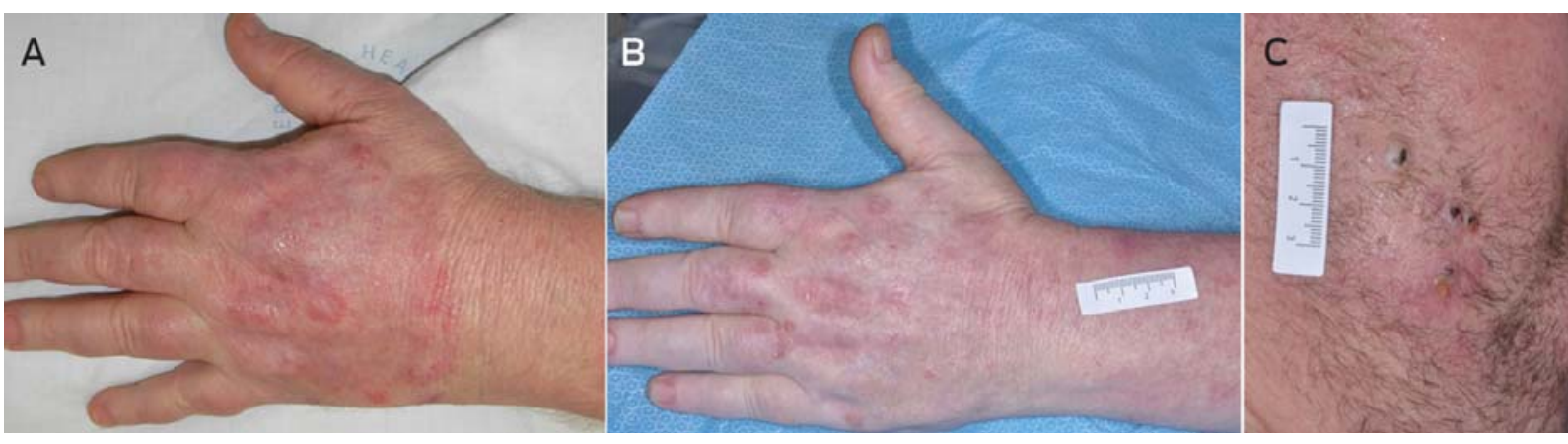

Rachael Anforth BMed $^{1,2}$

Giuliana Carlos MBBS $^{1,2}$

Noemi Eiris $\mathrm{MBBS}^{3}$

Arthur Clements BSc(Med), MBBS, FRACP ${ }^{1,2}$

Pablo

Fernandez-Peñas

$\mathrm{MD}, \mathrm{PhD}, \mathrm{FACD}$

1 Westmead Hospital, Sydney, NSW

2 University of Sydney,

Sydney, NSW.

3 Complejo Asistencial de León, León, Spain.

rachael.anforth@ sydney.edu.au

doi: 10.5694/mjal4.00848
$\mathrm{D}$ ermatophytic infections are usually located on the outermost layer of the epidermis. ${ }^{1}$ However, dermatophytes may affect deeper areas of the skin by invading hair follicles. When this happens, usually through a disruption of the epidermal barrier after the infection of hair follicles, ${ }^{1,2}$ an inflammatory granulomatous reaction can occur.

Dermatophytic dermal invasion causing inflammatory infiltrates of neutrophils and the development of granulomatous lesions is known as Majocchi granuloma or nodular granulomatous perifolliculitis. ${ }^{2}$ The dermatophyte that is most commonly involved is T. rubrum, ${ }^{1}$ the source of the T. rubrum is usually a precedent superficial dermatophytic infection such as the initial tinea corporis in our patient. Clinical examination often shows inflammatory follicular-centred papules, pustules or nodules on hair-bearing skin, which might evolve into larger subcutaneous nodules or abscesses.

While Majocchi granuloma is sometimes found in healthy individuals, development of the granulomatous reaction depends on the effectiveness of the immune system against the pathogen. ${ }^{3}$ Glucocorticoids affect cell-mediated immunity, impairing the function of macrophages and neutrophils, and reducing $T$ helper 1-mediated immunity, which plays an important role in the complete resolution of fungal infections. ${ }^{4}$

BRAF inhibitors, such as vemurafenib, are novel drugs that target an important mutation that is present in about $50 \%$ of metastatic melanomas. They have secondary effects on skin, such as development of multiple cutaneous squamous cell carcinomas, verrucal keratoses and a variable degree of photosensitivity. ${ }^{5-8}$ Since no 
vemurafenib-related immunosuppressive effects have been reported to date for vemurafenib, ${ }^{9,10}$ it is unlikely to have played a role in the development of the dermatophytic infection, apart from the severe drug-induced photosensitivity. The immunosuppressive glucocorticoid therapy for the brain metastases and vemurafenibinduced photosensitivity effectively placed the patient at risk of progressive infection from a superficial tinea corporis to a more invasive infection such as Majocchi granuloma. Given the well documented association of vemurafenib and photosensitivity reactions, the diagnosis was initially confounded and further aggravated by the treatment of the ultraviolet A photosensitivity. The introduction of topical antifungals was too late to stop progression of the disease, and the patient eventually developed Majocchi granuloma and a facial kerion for which oral antifungal therapy was needed.

In our patient, multiple cutaneous pathological processes were present concurrently. While the cutaneous secondary effects of vemurafenib are well reported, it is important to consider alternative diagnoses when there
Lessons from practice

- While vemurafenib induces ultraviolet A photosensitivity, it is important to consider alternative diagnoses when minimal response to therapy is attained.

- Concurrent administration of topical or systemic corticosteroids can alter the presentation of vemurafenib-induced adverse effects.

- Trichophyton rubrum can penetrate hair follicles to cause dermal infections that do not respond to topical therapy.

is minimal response to therapy. Immunosuppression from corticosteroid therapy (oral or topical) should be taken into consideration in patients taking these medications while also on antineoplastic therapies. Prompt recognition, diagnosis and treatment in this setting could avoid development of more serious complications.

Acknowledgement: Rachael Anforth has received a scholarship from the University of Sydney.

Competing interests: Pablo Fernandez-Peñas has received consultancy fees from Roche.

References are available online at www.mja.com.au. 
1 Sobera JO, Elewski BE. Fungal diseases. In: Bolognia JL, Jorizzo JL, Rapini RP, editors. Dermatology. 2nd ed. New York: Mosby Elsevier, 2008: 1135-1149.

2 Smith KJ, Welsh M, Skelton H. Trichophyton rubrum showing deep dermal invasion directly from the epidermis in immunosuppressed patients. Br J Dermatol 2001; 145: 344-348.

3 Burg M, Jaekel D, Kiss E, Kliem V. Majocchi's granuloma after kidney transplantation. Exp Clin Transplant 2006; 4: 518-520.

4 Azad A, Kaufman M, Jayarajan J. Dexamethasone-induced flares of Trichophyton rubrum masquerading as docetaxel cutaneous toxicity: a case report. Cases J 2009; 2: 8552.

5 Lemech C, Arkenau HT. Novel treatments for metastatic cutaneous melanoma and the management of emergent toxicities. Clin Med Insights Oncol 2012; 6: 53-66.

6 Anforth R, Fernandez-Peñas P, Long GV. Cutaneous toxicities of RAF inhibitors. Lancet Oncol 2013; 14: ell-el8.
7 Anforth RM, Blumetti TC, Kefford RF, et al. Cutaneous manifestations of dabrafenib (GSK2118436): a selective inhibitor of mutant BRAF in patients with metastatic melanoma. Br J Dermatol 2012; 167: 1153-1160.

8 Dummer R, Rinderknecht J, Goldinger SM. Ultraviolet A and photosensitivity during vemurafenib therapy. NEngl J Med 2012; 366: 480-481.

9 Hong DS, Vence L, Falchook G, et al. BRAF(V600) inhibitor GSK2118436 targeted inhibition of mutant BRAF in cancer patients does not impair overall immune competency. Clin Cancer Res 2012; 18: 2326-2335.

10 Knight DA, Ngiow SF, Li M, et al. Host immunity contributes to the anti-melanoma activity of BRAF inhibitors. J Clin Invest 2013; 123: 1371-1381 